bestehenden und bezogen auf die grundlegenden Entscheidungen auch nicht abwälzbaren Regelungsverantwortung ist der parlamentarische Gesetzgeber in Deutschland bisher bezogen auf den (absehbaren) Fall einer Pandemie nicht hinreichend nachgekommen. Wird er nicht bald tätig, „werden Kranke, Ärzte und Richter seine Feigheit ausbaden müssen“163.

163) Walter (Fn. 33).

\title{
Die COVID-19-Pandemie: Arbeitsrecht im Krankenhaus in bewegten Zeiten
}

\section{Alexander Eufinger}

\section{Einführung}

Nach nahezu einhelliger Meinung ist die COVID-19-Pandemie die größte Herausforderung an die Welt seit dem Zweiten Weltkrieg ${ }^{1}$. Dies gilt besonders für die Stabilität des Gesundheitssystems, aber auch für das gesellschaftliche und soziale Zusammenleben sowie die wirtschaftliche Stabilität und das Arbeitsleben. Auch in Deutschland ist jeder Arbeitgeber und Arbeitnehmer - zumindest mittelbar von dieser Pandemie betroffen. Die täglichen Betriebsabläufe werden durch das Corona-Virus SARS-CoV-2 grundlegend verändert. Dies gilt natürlich in besonderem Maße für die Tätigkeit im Krankenhaus, kämpfen doch deren Mitarbeiter an vorderster „Front“ gegen die COVID19-Pandemie und sehen sich einer immer noch nicht genau absehbaren Zahl infizierter Patienten gegenüber.

Erhebliche Auswirkungen des Corona-Virus SARSCoV-2 auf die Arbeitsverhältnisse von Ärzten, Pflegekräften und sonstigen medizinischen Fachangestellten sind daher unvermeidbar. Zwar wirkt die Klärung von arbeitsrechtlichen Fragestellungen vor dem Hintergrund der historischen Ausmaße der Corona-Krise nicht prioritär. Gleichwohl ergeben sich in dieser Situation für die im Krankenhaus Verantwortlichen eine Vielzahl von individualrechtlichen, aber auch betriebsverfassungsrechtlichen Fragestellungen und Problemen. So steht der Arbeitgeber „Krankenhaus“ hierbei vor einer besonders großen Herausforderung: auf der einen Seite muss er die Versorgung der Bevölkerung mit medizinischen Dienstleistungen gewährleisten und die Herausforderungen der COVID-19-Pandemie meistern, auf der anderen Seite hat er Sorge dafür zu tragen, dass die Erbringung der Arbeitsleistung ohne eine Gesundheitsgefährdung der Arbeitnehmer erfolgen kann. Auch in Zeiten der COVID-19-Pandemie ist die arbeitgeberseitige Fürsorgepflicht - besonders im Gesundheitswesen - nicht dispensiert. Gelingt den Krankenhäusern dieser Spagat zwischen dem Schutz der Mitarbeiter und der ausreichenden Versorgung der Patienten nicht, droht der befürchtete Kollaps des Gesundheitswesens.

Der nachstehende Beitrag gibt einen Überblick über die arbeitsrechtlichen Rahmenbedingungen, die ein Krankenhaus auch in diesen bewegten Zeiten beachten sollte. Im Mittelpunkt stehen hierbei die (vorübergehende) Änderung der Arbeitszeiten inklusive der Einführung von neuen Schichtmodellen und der sog. Kohortierung von Mitarbeitern sowie der Umgang mit einer zunehmenden

Dr. iur. Alexander Eufinger,

Leiter Geschäftsbereich Personal \& Recht,

Stiftung Hospital zum Heiligen Geist,

Steinbacher Hohl 2-26, 60488 Frankfurt, Deutschland
Zahl von Quarantänefällen und infiziertem medizinischen Personal. Zudem sollen flexible Urlaubsregelungen und arbeitsschutzrechtliche Fragestellungen thematisiert werden. Auch wenn die bestehende Gesetzeslage keinesfalls im Sinne einer Corona-Notstandslage ausgehebelt werden darf, sollen Anregungen für eine ,virusfreundliche“ ${ }^{\text {" }}$ - und damit pragmatische - Auslegung thematisiert werden.

\section{Arbeitszeitrecht}

\section{Neue Schichtmodelle}

Aufgrund der COVID-19-Pandemie und dem damit verbundenen Anstieg der zu behandelnden Patienten arbeitet die Mehrheit des medizinischen Personals im Krankenhaus oftmals über der Belastungsgrenze. Dies gilt sowohl für die psychische bzw. physische Belastung, als auch für die nach dem Arbeitszeitgesetz (ArbZG) vorgegebene Belastungsgrenze. Traditionell ist das Schichtsystem im Krankenhaus dreiteilig in Früh-, Spät- und Nachtdienst aufgeteilt. Dies führt zu einer Arbeitszeit von jeweils 7,7 oder 8 Stunden für den jeweiligen Mitarbeiter, was den Vorgaben des $\$ 3$ ArbZG entspricht, wonach die werktägliche Arbeitszeit der Arbeitnehmer acht Stunden nicht überschreiten darf. Überstunden sind hierbei durchaus möglich, kann doch die werktägliche Arbeitszeit auf bis zu zehn Stunden verlängert werden, wenn innerhalb von sechs Kalendermonaten oder innerhalb von 24 Wochen im Durchschnitt acht Stunden werktäglich nicht überschritten werden ( $\$ 3$ ArbZG). Im Zuge der COVID-19-Pandemie stellen jedoch viele Krankenhäuser ihre Schichtmodelle auf 12-Stunden-Schichtsysteme um. Zudem sollen die Pflegekräfte und Ärzte hierbei in festen Teams verbleiben, d.h. jeder Mitarbeiter arbeitet jeden Tag mit denselben Kollegen zusammen. Bei Versorgung von mehreren Fällen mit COVID-19 wird somit eine organisatorische und räumliche Trennung von Patienten und dem zugewiesenen Personal in einem gesonderten Bereich erreicht (sog. Kohortierung bzw. Kohortenbildung). Ziel ist es, die Kontaktdauer zwischen den einzelnen Kollegen und zwischen Mitarbeitern und Patienten zu begrenzen und so die Kontakthäufigkeit insgesamt zu verringern. Dies entspricht den Vorgaben des Robert Koch-Instituts, wonach medizinisches Personal, das in die Versorgung von Patienten mit COVID-19 Diagnose eingesetzt wird, möglichst von der Versorgung anderer Patienten freizustellen ist ${ }^{3}$. Infiziert sich

1) So etwa der UN-Chef António Guterres und Bundeskanzlerin Angela Merkel.

2) Zum Begriff vgl. Fuhlrott/Fischer, NZA 2020, 345.

3) www.rki.de/covid-19-hygiene. 
ein Mitarbeiter selbst mit dem Corona-Virus SARS-CoV-2, ist der potenzielle Kreis der von ihn kontaktierten Kollegen auf die jeweilige Kohorte begrenzt. Solange es geht, sollten jedoch die normalen, bisherigen Arbeitszeiten eingehalten werden, damit für den späteren befürchteten Ansturm infizierter Patienten ausreichend erholtes Personal zur Verfügung steht. Die tatsächliche Umstellung auf das 12-StundenSchichtsystem sollte erfolgen, wenn im Landkreis oder der Stadt der Notfall- bzw. Katastrophenplan ausgerufen wird oder wenn durch den täglichen Abgleich von Belegung und Schichtbesetzung deutlich wird, dass die 3-Schichtplanung durch Ausfälle nicht mehr funktioniert.

\section{Notfall oder außergewöhnlicher Fall nach $\$ 14$ Abs. 1 ArbZG}

Derartige Abweichungen von der gesetzlichen Höchstarbeitszeit von acht bzw. zehn Stunden nach $\$ 3$ ArbZG sind gemäß $₫ 14$ Abs. 1 ArbZG oder $\$ 15$ Abs. 2 ArbZG zulässig. So darf bei vorübergehenden Arbeiten in Notfällen und in außergewöhnlichen Fällen, die unabhängig vom Willen der Betroffenen eintreten und deren Folgen nicht auf andere Weise zu beseitigen sind, von den Vorgaben des $\$ 3$ ArbZG abgewichen werden. Unter einem Notfall im Sinne dieser arbeitszeitrechtlichen Ausnahmebestimmungen ist ein für den Betrieb widriges, ungewöhnliches, unvorhergesehenes und vom Willen des Betroffenen unabhängig und plötzlich eintretendes Ereignis zu verstehen, das die Gefahr eines unverhältnismäßigen Schadens mit sich bringt ${ }^{4}$. Demgegenüber brauchen außergewöhnliche Fälle keine Notfälle zu sein, sie müssen jedoch den Rahmen des Üblichen und Normalen überschreiten, unabhängig vom Willen des Betroffenen eintreten, und ihre Folgen müssen auf andere Weise nicht zu beseitigen sein. Außergewöhnlich ist ein Fall aber nur dann, wenn die Umstände, welche die Mehrarbeit bedingen, weder regelmäßig eintreten noch voraussehbar $\operatorname{sind}^{5}$. Epidemiologische Notlagen wie die der COVID19-Pandemie stellen sowohl einen Notfall, als auch einen außergewöhnlichen Fall i.S.d. $\$ 14$ Abs. 1 ArbZG dar. Es handelt sich um Umstände, die weder regelmäßig eintreten noch voraussehbar sind ${ }^{6}$. Voraussetzung ist jedoch auch in diesem Zusammenhang, dass das betreffende Krankenhaus ganz konkret von der COVID-19-Pandemie betroffen ist. Befindet sich das Krankenhaus in einem sog. Corona-Hotspot $^{7}$, wird ein besonderer Fall des $\$ 14$ ArbZG zu bejahen sein. Ist die Klinik bisher von einem größeren Ansturm infizierter Patienten verschont geblieben, liegt auch (noch) kein Notfall oder außergewöhnlicher Fall des $\$ 14$ ArbZG vor. Ohnehin ist in einer solchen Konstellation eine Umstellung auf das 12 -Stunden-Schichtsystem personalwirtschaftlich nicht angezeigt. Zudem dürfen Arbeitnehmer in Notfällen und außergewöhnlichen Fällen nur mit vorübergehenden Arbeiten beschäftigt werden. Hierunter sollen Arbeiten zu verstehen sein, die nicht allzu viel Zeit in Anspruch nehmen ${ }^{8}$. Die Spanne soll hierbei von wenigen Stunden bis hin zu mehreren Tagen reichen ${ }^{9}$. Im Rahmen der COVID-19-Pandemie werden wohl auch mehrere Wochen als ,,vorübergehend“ zu qualifizieren sein.

Darüber hinaus darf bei unaufschiebbaren Arbeiten zur Behandlung, Pflege und Betreuung von Personen von $\$ 3$ ArbZG abgewichen werden ( $\$ 14$ Abs. 2 Nr. 2 ArbZG). Zulässig sind somit Arbeiten zur Vermeidung von Gesundheitsstörungen, Untersuchungen, Krankentransporte, ambulante Behandlungen, Operationen, Versorgung etc ${ }^{10}$. Allerdings gestattet $₫ 14$ Abs. 2 Nr. 2 ArbZG die Beschäftigung expressis verbis nur an einzelnen Tagen. Zulässig soll daher die Beschäftigung an mehreren Tagen hintereinander, nicht aber über Wochen oder gar Monate sein ${ }^{11}$. Vereinzelt wird vertreten, dass die Ausnahme nicht mehr als 60 Tage im Jahr in Anspruch genommen werden darf ${ }^{12}$. Gegen diese teleologische Reduktion des $\$ 14$ Abs. 2 Nr. 2 ArbZG spricht der Wortlaut der Norm ${ }^{13}$, sodass auch bei einer häufigeren In- anspruchnahme als an 60 Tagen im Jahr, der Ausnahmetatbestand des $₫ 14$ Abs. 2 Nr. 2 ArbZG erfüllt sein wird ${ }^{14}$. Die vorübergehende Einführung eines 12-Stunden-Schichtsystems im Krankenhaus im Zuge der COVID-19-Pandemie wird vor diesem Hintergrund auch nach $\$ 14$ Abs. 2 Nr. 2 ArbZG gerechtfertigt sein. Sowohl bei $\$ 14$ Abs. 1 ArbZG, als auch nach $\int 14$ Abs. 2 Nr. 2 ArbZG ist jedoch zu beachten, dass die Arbeitszeit 48 Stunden wöchentlich im Durchschnitt von sechs Kalendermonaten oder 24 Wochen nicht überschreiten darf ( $\$ 14$ Abs. 3 ArbZG).

\section{Ausnahmegenehmigung im öffentlichen Interesse nach $\int 15$ Abs. 2 ArbZG}

Liegt ein außergewöhnlicher Fall oder Notfall i.S.d. \14 ArbZG vor, darf im 12-Stunden-Schichtsystem gearbeitet werden, wobei das Risiko falscher Einschätzungen der Arbeitgeber trägt ${ }^{15}$. Krankenhäuser sollten daher verfolgen, ob die jeweilige Aufsichtsbehörde eine Ausnahmebewilligung im öffentlichen Interesse nach $\$ 15$ Abs. 2 ArbZG erlassen hat. Die Aufsichtsbehörde kann über die im Gesetz vorgesehenen Ausnahmen hinaus weitergehende Ausnahmen zulassen, wenn ein dringendes öffentliches Interesse vorliegt. Ein öffentliches Interesse setzt ein dringendes Bedürfnis der Bevölkerung voraus. Hierzu gehört insbesondere das Erbringen von Dienstleistungen zur Gewährleistung der Daseinsvorsorge, wie die Energie- und Wasserversorgung, die Abwasser- und Abfallentsorgung, Polizei und natürlich der Krankenhausbetrieb sowie die Pflegebranche ${ }^{16}$. Mittlerweile haben nahezu alle Regierungspräsidien bzw. Bezirksregierungen Ausnahmebewilligungen für derartige Abweichungen von bestimmten Beschränkungen des ArbZG aus Anlass der Ausbreitung des Corona-Virus SARS-CoV-2 in Deutschland gemäß $\$ 15$ Abs. 2 ArbZG erlassen. Insofern wird sich ein Krankenhaus bei der Einführung eines 12-Stunden-Schichtsystems regelmäßig auf rechtssicherem Terrain bewegen. Allerdings darf auch mit der Ausnahmegenehmigung des $\$ 15$ Abs. 2 ArbZG die Arbeitszeit 48 Stunden wöchentlich im Durchschnitt von sechs Kalendermonaten oder 24 Wochen nicht überschreiten.

\section{Mitbestimmungsrechte und Schichtmodelle}

Der Betriebsrat hat bei der Einführung neuer Schichtmodelle - insbesondere eines 12-Stunden-Schichtsystems - ein Mitbestimmungsrecht nach $₫ 87$ Abs. 1 Nr. 2 BetrVG. Bei der Ausgestaltung von Schichtarbeit erfasst das Mitbestimmungsrecht aus $\int 87$ Abs. 1 Nr. 2 BetrVG nicht nur die Frage, ob im Betrieb in mehreren Schichten gearbeitet werden soll, sondern auch die Festlegung der zeitlichen Lage der einzelnen Schichten und die Abgrenzung des Personenkreises, der Schichtarbeit zu leisten hat. Mitbestimmungspflich-

4) OLG Hamburg, AP AZG $₫ 8$ Nr. 1

5) BAG, BeckRS 1986, 30718337.

6) So auch Fuhlrott/Fischer, NZA 2020, 345, 347

7) Bekanntestes Beispiel für einen solchen Hotspot war zu Beginn der COVID-19-Pandemie der Landkreis Heinsberg.

8) Wank, in: ErfK, 20. Aufl. 2020, \$14 ArbZG, Rdnr. 4.

9) Neumann/Biebl, ArbZG, 16. Aufl. 2012, \14, Rdnr. 6; Baeck/ Deutsch, ArbZG, 3. Aufl. 2014, $\$ 14$, Rdnr. 16.

10) Baeck/Deutsch, ArbZG, 3. Aufl. 2014, $\$ 14$, Rdnr. 41.

11) Wank, in: ErfK, 20. Aufl. 2020, $\$ 14$ ArbZG, Rdnr. 9.

12) Anzinger/Koberski, ArbZG, 4. Aufl. 2014, $\$ 14$, Rdnr. 21.

13) Neumann/Biebl, ArbZG, 16. Aufl. 2012, \$14, Rdnr. 17.

14) Anzinger/Koberski, ArbZG, 4. Aufl. 2014, \$14, Rdnr. 21

15) Fuhlrott/Fischer, NZA 2020, 345, 347

16) So zum Beispiel explizit die Bezirksregierung Köln, Ausnahmebewilligung zur Beschäftigung von Arbeitnehmerinnen und Arbeitnehmern gemäß $\$ 15$ Abs. 2 Arbeitszeitgesetz (ArbZG) aus Anlass der Ausbreitung des Corona-Virus SARS-CoV-2 sowie der dadurch hervorgerufenen Erkrankung COVID-19 in Deutschland. 
tig ist auch der Schichtplan und dessen nähere Ausgestaltung bis hin zur Zuordnung der Arbeitnehmer zu den einzelnen Schichten ${ }^{17}$. Die COVID-19-Pandemie stellt auch viele Betriebsräte in Krankenhäusern vor besondere Herausforderungen: einerseits ist das Kollektivorgan bemüht, die von ihm vertretene Belegschaft vor einer erhöhten Arbeitsbelastung $^{18}$ und ,italienischen“ Corona-Verhältnissen zu schützen, andererseits wird die historische Dimension bei der Bekämpfung der COVID-19-Pandemie nicht verkannt. Besonders in der Corona-Krise kommt dem Primat der vertrauensvollen Zusammenarbeit nach $\$ 2$ Abs. 1 BetrVG, wonach Arbeitgeber und Betriebsrat unter Beachtung der geltenden Tarifverträge vertrauensvoll zum Wohl der Arbeitnehmer und des Betriebs zusammenarbeiten sollen, eine herausragende Bedeutung zu. So darf die Krankenhausleitung die COVID-19-Pandemie nicht dazu ausnutzen, Betriebsräte ausschließlich unter Druck zum Abschluss einer 12-Stunden-Schichtsystem-Betriebsvereinbarung zu bewegen. Krankenhausbetriebsräte sind im Gegenzug aber auch verpflichtet, die COVID-19-Pandemie nicht zur Durchsetzung bereits langgehegter Wünsche auszunutzen. Daher sollte vereinbart werden, dass alle bisherigen Betriebs- oder Dienstvereinbarungen zur Arbeitszeit und Schichtmodellen ihre Gültigkeit behalten und durch eine zusätzliche Vereinbarung entsprechende Sonderregelungen in Form des 12-Stunden-Schichtsystems nur für einen befristet festgelegten Zeitraum gelten. Dieser Zeitraum sollte nach Möglichkeit ebenfalls möglichst kurz definiert werden, damit je nach epidemiologischer Krisenlage und Patientenanfall neu entschieden werden kann. Auch die Ausnahmetatbestände nach $\$ \$ 14,15$ ArbZG wurden geschaffen, um besondere Krisensituationen zu bestehen und nicht dauerhaft auf - für die Beschäftigten - belastende Dienstmodelle umzusteigen. Wichtig ist zudem, ein Mitglied des Betriebsrates (möglichst den Betriebsratsvorsitzenden) in den krankenhauseigenen Corona-Krisenstab einzubeziehen - auch wenn auf diese Teilnahme kein betriebsverfassungsrechtlicher Anspruch besteht. Auf diese Weise kann gewährleistet werden, dass Entscheidungen des Corona-Krisenstabes - regelmäßig bestehend aus kaufmännischen, ärztlichen sowie pflegerischen Führungskräften - für den Betriebsrat transparent und nachvollziehbar sind. Zudem kann das Betriebsratsmitglied selbst konstruktive Vorschläge im Krisenstab einbringen.

\section{Kurzarbeit}

a) Sozialversicherungsrechtliche Voraussetzungen

Anders als von der breiten Öffentlichkeit - aber auch von juristischen Fachkreisen ${ }^{19}$ - vermutet, wird auch in vielen Krankenhäusern derzeit über Kurzarbeit diskutiert. Zumindest in einem Unternehmen der Gesundheitsversorgung handelt es sich in Zeiten der COVID-19-Pandemie bei dieser Diskussion eigentlich um eine Absurdität sondergleichen. Hintergrund ist, dass zu Beginn der COVID-19-Pandemie alle Krankenhäuser von der Bundes- sowie den jeweiligen Landesregierungen dazu verpflichtet wurden, planbare, medizinisch nicht zwingend notwendige Operationen abzusagen, um die Kliniken auf Corona-Patienten vorzubereiten ${ }^{20}$. Krankenhäusern ist damit buchstäblich von heute auf morgen ein Großteil des operativen Geschäfts weggebrochen. Insbesondere in chirurgischen Bereichen stellte bzw. stellt sich die Frage, wie Mitarbeiter nach Überstundenabbau und dem Nehmen von Resturlaub vertragsgemäß beschäftigt werden können, solange der Höhepunkt der COVID19-Pandemie dieses Krankenhaus noch nicht erreicht hat. Grundsätzlich kann auch ein Krankenhaus Kurzarbeitergeld bei der Bundesagentur für Arbeit beantragen ${ }^{21}$. Hierfür müssen die Voraussetzungen der $\$ \int 95$ bis 99 SGB III vorliegen. Der Anspruch besteht, wenn ein erheblicher Arbeitsausfall vorliegt, die betrieblichen sowie persönlichen Voraussetzungen erfüllt sind und der Arbeitsausfall angezeigt worden ist.
Der Arbeitsausfall muss auf wirtschaftlichen Gründen oder einem unabwendbaren Ereignis beruhen, vorübergehend und nicht vermeidbar sein und im jeweiligen Monat eine Erheblichkeitsschwelle überschreiten (vgl. \96 Abs. 1 S. 1 SGB III). Das Merkblatt „Kurzarbeitergeld (KUG): CoronaVirus: Informationen für Unternehmen" der Bundesagentur für Arbeit sieht vor, dass Arbeitnehmer Kurzarbeitergeld erhalten können, wenn aufgrund der COVID-19-Pandemie Aufträge ausbleiben oder storniert werden oder wenn aufgrund staatlicher Schutzmaßnahmen Arbeit entfällt. Aufgrund der behördlich angeordneten Aussetzung elektiver Eingriffe und geplanter Behandlungen brechen Krankenhäusern wichtige Einnahmen ${ }^{22}$ weg - bei gleichzeitig fehlender Kompensation über COVID-19-infizierte Patienten, ist doch nicht jede Klinik in Deutschland gleichmäßig von der COVID-19-Pandemie betroffen ${ }^{23}$. Die Mindestanforderungen an die Erheblichkeit des Arbeitsausfalls benennt $\$ 96$ Abs. 1 Nr. 4 SGB III. Gemäß $\$ 1$ Nr. 1 der am 23.3.2020 erlassenen Kurzarbeitergeldverordnung (KugV) gilt rückwirkend zum 1.3.2020, dass ein Betrieb Kurzarbeit anmelden kann, wenn mindestens zehn Prozent der Beschäftigten vom Arbeitsausfall betroffen sind. Diese Schwelle lag bisher bei einem Drittel der Belegschaft. Gelockert wurden auch die Anforderungen an die Unvermeidbarkeit des Arbeitsausfalls in $\$ 96$ Abs. 4 SGB III. So ist der Aufbau negativer Arbeitszeitsalden (,Minusstunden“) vor Zahlung des Kurzarbeitergeldes nicht mehr erforderlich ( $\$ 1 \mathrm{Nr} .2 \mathrm{KugV})$. Die betrieblichen Voraussetzungen erfüllt jeder Betrieb, in dem mindestens ein Arbeitnehmer beschäftigt ist. Persönlich erfüllt ein Arbeitnehmer die Voraussetzungen für den Bezug von Kurzarbeitergeld nach $\$ 98$ Abs. 1 SGB III, wenn er in einem ungekündigten, versicherungspflichtigen Arbeitsverhältnis steht. Vom Kurzarbeitergeld ausgenommen sind demnach geringfügig Beschäftigte, wenn sie nicht versicherungspflichtig sind. Hingegen können befristet Beschäftigte Kurzarbeitergeld erhalten. Die für Arbeitgeber anfallenden Beiträge zur Sozialversicherung für ausgefallene Arbeitsstunden werden auf Antrag von der Agentur für Arbeit zu $100 \%$ in pauschalierter Form erstattet (\$2 KugV).

\section{b) Arbeitsrechtliche Voraussetzungen}

Neben den Voraussetzungen der $\$ \int 95$ bis 99 SGB III müssen auch die arbeitsrechtlichen Voraussetzungen zur Einführung von Kurzarbeit vorliegen. So ist der Arbeitgeber nicht zur einseitigen Einführung von Kurzarbeit berechtigt. Das Direktionsrecht des Arbeitgebers ist nicht ausreichend. Vielmehr bedarf er hierzu einer besonderen rechtlichen Grundlage ${ }^{24}$. In vielen Branchen existieren tarifliche Regelungen, die unter bestimmten näher definierten Voraussetzungen die Einführung von Kurzarbeit

17) BAG, NZA 2014, 99, 100

18) Ahrendt, in: Schaub, Arbeitsrechts-Handbuch, 18. Aufl. 2019, $\$ 235$, Rdnr. 42.

19) Vgl. Fuhlrott/Fischer, NZA 2020, 345

20) So z.B. für das Land Hessen: „Fünfte Verordnung zur Bekämpfung des Corona-Virus v. 16.3.2020“. In dieser Verordnung des Hessischen Ministeriums für Soziales und Integration heißt es: „Die Durchführung von medizinischen Eingriffen und Behandlungen, für die derzeit keine dringende medizinische Notwendigkeit besteht (nicht notwendige Behandlungen), wird in folgenden Einrichtungen ausgesetzt: [...] Krankenhäuser [...]“.

21) Ebenfalls relevant ist der mögliche Anspruch auf Kurzarbeitergeld auch für viele niedergelassene Ärzte in eigener Praxis; so leiden auch Haus-, aber insbesondere auch Fachärzte sowie Medizinische Versorgungszentren unter Absagen langfristig geplanter Termine; vgl. ÄrzteZeitung v. 20.3.2020.

22) Also letztlich Aufträge i.S.d. \$96 Abs. 1 S. 1 SGB III.

23) FAZ v. 22.3.2020: „Verband über COVID-19-Gesetz: Totenglocken für Kliniken“; sowie FAZ v. 19.3.2020: „Corona: Krankenhäuser befürchten Pleitewelle".

24) BAG, NZA 2009, 689, 691. 
ermöglichen. In Zeiten von pflegerischem und ärztlichem Personalmangel sowie der Einführung von Pflegepersonaluntergrenzen sieht der Tarifvertrag für den öffentlichen Dienst für den Dienstleistungsbereich Krankenhäuser (TVöD-K) bezeichnenderweise keine tarifliche Ermächtigungsgrundlage für die Einführung von Kurzarbeit vor ${ }^{25}$ Nach $\$ 87$ Abs. 1 Nr. 3 BetrVG hat der Betriebsrat bei der vorübergehenden Verkürzung oder Verlängerung der betriebsüblichen Arbeitszeit mitzubestimmen. Hierunter fällt die Einführung von Kurzarbeit, und zwar auch dann, wenn Tage oder Wochen endgültig ausfallen und damit die Dauer der Arbeitszeit berührt wird ${ }^{26}$. Als Rechtsgrundlage für die Einführung von Kurzarbeit kann daher in Betrieben mit Betriebsrat eine Betriebsvereinbarung dienen. Ohne Rücksicht auf den Willen der betroffenen Arbeitnehmer kann durch sie grundsätzlich eine Änderung der Arbeitsbedingungen auch hinsichtlich der Arbeitszeit und der Lohnzahlungspflichten erfolgen ${ }^{27}$. In betriebsratslosen Betrieben kann den Arbeitgeber nur eine einzelvertragliche Vereinbarung zur Einführung von Kurzarbeit berechtigen. Diese kann entweder aus konkretem Anlass oder bereits bei Abschluss des Arbeitsvertrages getroffen werden.

\section{Lohnfortzahlung in Zeiten der COVID-19-Pandemie}

\section{Erkrankung des Arbeitnehmers}

\section{a) Grundsatz der Entgeltfortzahlung}

Erleidet der Arbeitnehmer eine Erkrankung durch das Corona-Virus SARS-CoV-2 und ist er infolgedessen arbeitsunfähig, steht ihm wie bei jeder krankheitsbedingten Arbeitsunfähigkeit ein Anspruch auf Entgeltfortzahlung durch den Arbeitgeber nach $\$ 3$ Abs. 1 EFZG zu. D.h., der Arbeitnehmer erhält in diesem Fall vom Arbeitgeber die vereinbarte Vergütung in voller Höhe, die er bei normaler Ausübung seiner Tätigkeit, ohne krank gewesen zu sein, erhalten hätte ( $\$ 4$ Abs. 1 EFZG). Die Dauer der Entgeltfortzahlung erstreckt sich auf einen Zeitraum von sechs Wochen und beginnt mit dem ersten Tag der Erkrankung. Hierbei kann der Zeitraum der Entgeltfortzahlung aber auch mehr als sechs Wochen betragen, insofern sich eine abweichende Regelung aus Arbeits- oder Tarifvertrag ergibt. Allerdings erfolgt eine Begrenzung der Lohnfortzahlung dahingehend, dass im Zeitraum der Erkrankung eigentlich anfallende Ansprüche zur Vergütung von Überstunden - im Gesundheitswesen aus praktischer Sicht sehr relevant - nach $\ 4$ Abs. 1a EFZG unberücksichtigt bleiben.

b) Verschulden gegen sich selbst

Die Geltendmachung eines Entgeltfortzahlungsanspruchs setzt nach $\$ 3$ Abs. 3 EFZG voraus, dass das Arbeitsverhältnis bereits seit einer ununterbrochenen Dauer von vier Wochen Bestand hat und den Arbeitnehmer hinsichtlich des Eintretens der Arbeitsunfähigkeit kein Verschulden trifft. Ergeben sich für den Arbeitgeber Anhaltspunkte, die das Vorliegen einer Infektion mit dem Corona-Virus SARSCoV-2 aufgrund eines schuldhaften Verhaltens des Arbeitnehmers nahelegen, könnte die Verweigerung der Entgeltfortzahlung zu prüfen sein. Hierfür ist der nach gefestigter Rechtsprechung des BAG eigens für die Entgeltfortzahlung entwickelte Verschuldensmaßstab anzulegen. Danach ist ein Verschulden des Arbeitnehmers im Sinne des EFZG anzunehmen, wenn dieser, ,in erheblichem Maße gegen die von einem verständigen Menschen im eigenen Interesse $\mathrm{zu}$ erwartende Verhaltensweise verstößt $\mathrm{t}^{\text {"28 }}$. Insofern wird auch ausdrücklich von einem ,Verschulden gegen sich selbst" gesprochen ${ }^{29}$. Dieses liegt vor, wenn das Verhalten des Arbeitnehmers als besonders leichtfertig oder vorsätzlich einzustufen ist. Bei der Ergründung des Verschuldens ist auf einen objektiven Maßstab abzustellen. In der
Praxis haben die Arbeitsgerichte bislang insbesondere die schuldhafte Herbeiführung einer Arbeitsunfähigkeit von Arbeitnehmern im Rahmen von Unfällen bei der Arbeit, beim Sport oder im Straßenverkehr zu prüfen gehabt ${ }^{30}$. Die Darlegungs- und Beweislast hierfür trifft den Arbeitgeber, wenngleich der Arbeitnehmer zur Mitwirkung bei der Aufklärung der wesentlichen Umstände verpflichtet werden $\mathrm{kann}^{31}$. Ein Leistungsverweigerungsrecht des Arbeitgebers wird allerdings nur schwerlich zu bejahen sein. Dies betrifft denkbar seltene Fallkonstellationen, in denen etwa ein Arbeitnehmer in seinem Privatleben womöglich billigend oder gar vorsätzlich eine Infizierung in Kauf genommen hat, indem er sich beispielsweise dem staatlich angeordneten Kontaktverbot widersetzt hat ${ }^{32}$. Ein Ausschluss des Anspruchs nach $\$ 3$ EFZG kommt etwa auch in Betracht, wenn sich ein Arbeitnehmer leichtfertig oder vorsätzlich in ein vom Robert Koch-Institut qualifiziertes Risikogebiet begibt und dort mit dem Corona-Virus SARS-CoV-2 infiziert $^{33}$. Es ist Arbeitgebern daher anzuraten, eine Aussetzung der Entgeltfortzahlung lediglich dann in Erwägung $\mathrm{zu}$ ziehen, wenn ein deutlich offensichtliches Fehlverhalten für die eingetretene Infizierung mit dem Corona-Virus SARS-CoV-2 nahezuliegen scheint.

\section{Eigenmächtiges Fernbleiben von der Arbeit}

Gleichwohl sind Situationen denkbar, in denen Arbeitnehmer zwar noch nicht mit dem Corona-Virus SARS-CoV-2 infiziert sind, aber aus Vorsorge oder Angst vor einer Infektion zu Hause bleiben möchten, um einer Ansteckungsgefahr auf dem Weg zur Arbeit oder am Arbeitsplatz selbst zu entgehen. Allein der Ausbruch einer Pandemie rechtfertigt ein eigenmächtiges Fernbleiben des Arbeitnehmers von der Arbeitsstelle nicht, auch wenn die Ansteckungsgefahr am Arbeitsplatz durch den Kontakt mit anderen Menschen - insbesondere im Krankenhaus - deutlich erhöht ist ${ }^{34}$. Insofern verletzt der Arbeitnehmer seine gegenüber dem Arbeitgeber zu erbringende Arbeitspflicht, wenn er bei bloBer Angst vor einer Infektion seine Arbeitstätigkeit nicht aufnimmt. Der Arbeitgeber hat dann nach $₫ 320$ Abs. 1 BGB das Recht, die Vergütung einzubehalten ${ }^{35}$. Gleichzeitig steht es dem Arbeitgeber in diesem Fall frei, gegenüber dem Arbeitnehmer bei Nichterscheinen am Arbeitsplatz arbeitsrechtliche Sanktionen, im Wiederholungsfall bis hin zur Kündigung, auszusprechen ${ }^{36}$. Etwas anderes gilt nur dann, wenn der Arbeitnehmer nach $\$ 275$ Abs. 3 BGB zur Verweigerung der Arbeitsleistung berechtigt sein sollte. Ein solches Leistungsverweigerungsrecht setzt voraus, dass dem Arbeitnehmer die Erbringung der persönlichen Arbeitsleistung unter Abwägung des ihm seiner Leistung entgegenstehenden Hindernisses mit dem Leistungsinter-

25) Ebenfalls ist in den Tarifverträgen für Medizinische Fachangestellte (MFA) Kurzarbeit nicht geregelt.

26) BAG, NZA 1991, 607, 608.

27) BAG, NZA 2009, 689, 691

28) BAG, NZA 2017, 240; BAG, NZA 2015, 801.

29) BAG, NZA 2015, 801; Reinhard, in: ErfK, 20. Aufl. 2020, $\$ 3$ EFZG, Rdnr. 23.

30) Näher dazu Greiner, in: MHdB ArbR, 4. Aufl. 2018, $\$ 80$, Rdnrn. $59 \mathrm{ff}$.

31) Einführend hierzu Linck, in: Schaub, Arbeitsrechts-Handbuch, 18. Aufl. 2019, $\$ 98$, Rdnr. 42

32) Hierzu nähergehend beschäftigt Groeger, ArbRB-Blog v. 28.3.2020; sowie das praktische Beispiel eines Berliner Bezirksbürgermeisters, der sich absichtlich mit dem Corona-Virus SARS-CoV-2 infiziert hat, vgl. DIE WELT v. 1.4.2020.

33) Fuhlrott/Fischer, NZA 2020, 345, 347.

34) Vgl. Steinau-Steinrück/Mosch, NJW-Spezial 2009, 578; Kraft/Drohmen, PharmR 2008, 401, 405.

35) Schmidt/Novara, DB 2009, 1817, 1820

36) Bonanni, ArbRB 2020, S1. 
esse des Arbeitgebers nicht zugemutet werden kann. Bei Vorliegen einer derartigen Pflichtenkollision kann dem Arbeitnehmer die Erbringung der Arbeitsleistung unzumutbar sein, wenn dadurch die Verletzung anderer bedeutsamer Rechtsgüter in Gefahr stehen würde ${ }^{37}$. Eine mögliche Infizierung mit dem Corona-Virus SARS-CoV-2 begründet eine erhebliche Gefahr für Leben oder Gesundheit des Krankenhauspersonals. Vor diesem Hintergrund wird eine etwaige Unzumutbarkeit der Arbeitsleistung in bestimmten Ausnahmekonstellationen durchaus diskutiert ${ }^{38}$. Unabhängig von der aus medizinischer Perspektive schwierig zu beurteilenden Erheblichkeit der Gefahr durch das Virus wird die Erfüllung des Tatbestands des $\$ 275$ Abs. 3 BGB aber grundsätzlich zu verneinen sein, sofern das Krankenhaus die in der Situation erforderlichen und zumutbaren Schutzmaßnahmen veranlasst und gewährleistet (vgl. hierzu Punkt VI. $)^{39}$. Aus diesem Grund werden sich Ärzte oder Pflegekräfte im Hinblick auf das Rechtsgut der körperlichen Unversehrtheit nicht auf ein Leistungsverweigerungsrecht aufgrund eines bestehenden Gesundheitsrisikos berufen können ${ }^{40}$. Generell wird eine Unzumutbarkeit der persönlichen Leistungserbringung nur in absoluten Ausnahmefällen in Betracht zu ziehen sein, z. B. wenn der Arbeitnehmer aufgrund einschlägiger Vorerkrankungen oder Schwangerschaft möglicherweise einem erhöhten Risiko ausgesetzt ist ${ }^{41}$. Zudem ist darauf hinzuweisen, dass der Arbeitnehmer bei Geltendmachung des Leistungsverweigerungsrechts in Rechtsfolge des $\$ 275$ Abs. 3 BGB i. V.m. \326 Abs. 1 S. 1 BGB seines Vergütungsanspruches grundsätzlich verlustig wird ${ }^{42}$.

\section{COVID-19-Pandemie und Wegerisiko}

Daneben gilt für die Fahrt zum Arbeitsplatz, dass der Arbeitnehmer das Wegerisiko zu tragen hat ${ }^{43}$. Führt die Ausbreitung des Corona-Virus SARS-CoV-2 dazu, dass der Arbeitnehmer etwa aufgrund des Ausfalls des öffentlichen Nahverkehrs die Tätigkeit an seiner Arbeitsstätte nicht aufnehmen kann, ist der Arbeitgeber ebenso zur Einbehaltung des Vergütungsanspruchs berechtigt ${ }^{44}$. Ein Annahmeverzug der Arbeitsleistung durch den Arbeitgeber liegt schließlich nicht vor. Der Arbeitnehmer ist in dem Fall allerdings im Wege des $\$ 275$ Abs. 1 BGB von der Leistungspflicht befreit und braucht keine arbeitsrechtlichen Sanktionen zu fürchten. Dies ist anders zu beurteilen, wenn die Anfahrt zur Tätigkeitsstätte weiterhin möglich ist, der Arbeitnehmer aber aus bloßer Angst vor einer Infektion während des Anfahrtsweges die Tätigkeitsaufnahme verweigert. Eine Befreiung von der Arbeitspflicht ist selbst dann nicht anzunehmen, wenn die Behörden für die Nutzung von öffentlichen Verkehrsmitteln eine Warnung aussprechen würden ${ }^{45}$. Von weitaus größerer Relevanz war zumindest zu Beginn der COVID-19-Pandemie die Frage, wie mit Mitarbeitern umzugehen ist, die im Ausland - oftmals an ihrem Urlaubsort - festsaßen und an einer Rückreise in die Heimat gehindert waren. Auch hier gilt der Grundsatz des Wegerisikos für den Arbeitnehmer. Kann der Arbeitnehmer seinen Urlaubsort nicht mittels der üblichen Verkehrswege verlassen - z. B. weil die Fluggesellschaften im Zeichen der Corona-Krise sämtliche Flüge eingestellt haben -, um seinen Betrieb zur Erfüllung seiner Arbeitspflicht aufzusuchen, ist dem Arbeitnehmer die Erbringung der Arbeitsleistung unmöglich ${ }^{46}$. In diesem Fall ist der Arbeitnehmer von seiner Arbeitspflicht befreit, den Vergütungsanspruch verliert er allerdings auch (\$S 275 Abs. 1, 326 Abs. 1 BGB). Allerdings sollte der Arbeitnehmer der ihm aus dem Arbeitsverhältnis erwachsenen Pflicht nachkommen und die Arbeitsverhinderung dem Arbeitgeber unverzüglich anzeigen. Das weitere Vorgehen zur Bewältigung der Situation sollte dann zwischen Arbeitnehmer und Arbeitgeber einvernehmlich geklärt werden.

\section{Häusliche Quarantäne des Arbeitnehmers}

Nach den Vorgaben der $\$ \$ 28,30$ IfSG sind die zuständigen Behörden dazu berechtigt, bereits dann die Quarantäne für Personen anzuordnen, wenn diese lediglich in Kontakt zu Infizierten gestanden haben, ohne selbst erkrankt zu sein. Wird ein Arbeitnehmer ohne Anzeichen von einschlägigen Krankheitssymptomen allein vorsorglich aufgrund des Kontakts mit Infizierten in häusliche Quarantäne gestellt, wird er damit nicht automatisch von seiner Arbeitspflicht entbunden. Schließlich ist er (noch) nicht akut erkrankt. So wird die weitere Ausübung der Arbeitstätigkeit zumindest dann fortzusetzen sein, soweit dies im Homeoffice möglich ist. Kann der Arbeitnehmer seiner Tätigkeit im Homeoffice nicht nachgehen, was bei ärztlichem und pflegerischem Personal regelmäßig der Fall sein wird, ist dagegen nach $\$ 275$ Abs. 1 die Unmöglichkeit der Arbeitsleistung gegeben ${ }^{47}$. In diesem Fall liegt eine Befreiung von der Arbeitspflicht vor. Die Unmöglichkeit der Arbeitsleistung hat für den Betroffenen jedoch auch den Wegfall des Vergütungsanspruches gemäß \326 Abs. 1 BGB zur Folge. Ebenso wenig liegen die Voraussetzungen für einen Entgeltfortzahlungsanspruch nach $\$ 3$ EFZG vor. So ist eine für $\$ 3$ EFZG erforderliche Arbeitsunfähigkeit gerade nicht gegeben, da der Mitarbeiter lediglich zur Vorsorge aufgrund des Kontaktes zu einem oder mehreren Infizierten in Quarantäne beordert wurde, aber nicht selbst erkrankt ist. Allerdings greift hier die Regelung des $₫ 56$ IfSG. Diese sieht eine Entschädigung für den Arbeitnehmer in Höhe des Netto-Arbeitsentgelts für die ersten sechs Wochen der Quarantäne vor. Die Entschädigung zahlt der Arbeitgeber aus, bekommt sie aber auf Antrag von den zuständigen Behörden erstattet. Ab der siebten Quarantäne-Woche zahlen die zuständigen Behörden eine Entschädigung in Höhe des Krankengeldes direkt an den Arbeitnehmer. Eine Verrechnung mit seinem Jahresurlaub findet nicht statt. Die zuständigen Behörden vertreten zum Teil die Auffassung, dass der Arbeitgeber vorrangig verpflichtet sei, über $\int 616$ BGB das Entgelt fortzuzahlen. Dieser Anspruch setzt voraus, dass der Arbeitnehmer für eine verhältnismäßig nicht erhebliche Zeit durch einen in seiner Person liegenden Grund ohne sein Verschulden an der Dienstleistung verhindert wird. Mit Verweis auf die bisherige Rechtsprechung des BGH kann eine behördliche Maßnahme wie die Anordnung einer Quarantäne den Anspruch auf Entgeltzahlung für bis zu sechs Wochen rechtfertigen ${ }^{48}$. Angesichts der Tatsache, dass dieses Urteil des BGH mehr als 40 Jahre zurückliegt, ist nicht auszuschließen, dass dieser inzwischen anders entscheiden würde. Darüber hinaus sagt die Bundesregierung angesichts der Intensität der Wirtschaftskrise umfangreiche finanzielle Unterstützung zu. Vor diesem Hintergrund sollte bei behördlich veranlassten Quarantänemaßnahmen oder Beschäftigungsverboten auf jeden Fall ein Antrag auf Erstattung bei der zuständigen Behörde gestellt werden. Ungeachtet dessen, ist ein Anspruch auf Entgeltfortzahlung nach $\int 616$ BGB zumindest dann nicht einschlägig, wenn

37) Legerlotz, ArbRB 2017, 157, 158.

38) Vgl. Preis, in: ErfK, 20. Aufl. 2020, \$611a BGB, Rdnr. 686.

39) Schmidt/Novara, DB 2009, 1817, 1820.

40) Vgl. Preis, in: ErfK, 20. Aufl. 2020, \$611a BGB, Rdnr. 686; Legerlotz, ArbR B 2017, 157, 158.

41) Fuhlrott, GWR 2020, 107; Kraft/Drohmen, PharmR 2008, 401, 405.

42) Legerlotz, ArbRB 2017, 157, 159.

43) Preis, in: ErfK, 20. Aufl. 2020, $\$ 615$ BGB, Rdnr. 133.

44) Vgl. Schmidt/Novara, DB 2009, 1817, 1820.

45) So jedenfalls Schaer, MDR 2020, R5-R7.

46) Vgl. Mareck, AuA 2019, 034-035.

47) Weller/Lieberknecht/Habrich, NJW 2020, 1017, 1018.

48) BGH, NJW 1979, 422. 
dieser durch Arbeits- oder Tarifvertrag abbedungen wur$\mathrm{de}^{49}$. In diesem Fall verbleibt allein ein Rückgriff auf den staatlichen Entschädigungsanspruch nach $\$ 56$ IfSG.

\section{Management von Kontaktpersonen}

Im Hinblick auf die Sicherstellung der Patientenversorgung liegt es im Interesse des Krankenhausbetreibers, medizinisches Personal, welches sich aufgrund von Kontakt zu Infizierten in einer Quarantäne befindet, möglichst früh wieder für den Dienst einplanen zu können. Um diesem Zielkonflikt - zwischen einzuhaltenden Quarantänemaßnahmen auf der einen Seite und der Aufrechterhaltung der medizinischen Versorgung auf der anderen Seite - gerecht zu werden, hat das Robert Koch-Institut Empfehlungen zum Management von Kontaktpersonen unter medizinischem Personal bei Personalmangel veröffentlicht ${ }^{50}$. Danach kann der Umgang mit Kontaktpersonen unter medizinischem Personal bei Personalengpässen angepasst werden, sofern anderenfalls die Gewährleistung der Patientenversorgung gefährdet wäre. Dies ermöglicht einen effektiven Einsatz von medizinischem Personal. Vordergründig sind zunächst aber andere Handlungsoptionen zu prüfen, beispielsweise die Verlegung von Patienten in andere Kliniken oder die - nur schwerlich mögliche - Rekrutierung neuen Personals. Das Robert Koch-Institut clustert hierbei die Mitarbeiter in Kontaktpersonen der Kategorie I bis III und knüpft hieran verschiedene organisatorische Maßnahmen. Betont wird zudem die Bedeutung der Einhaltung der persönlichen Schutzausrüstung und Arbeitsschutzvorschriften (siehe Punkt VI.). An die Umsetzung dieser Empfehlungen sind die Verantwortlichen der medizinischen Einrichtungen nach $\$ 23$ Abs. 3 IfSG gebunden. Die Anweisung an in Quarantäne befindliche Mitarbeiter wieder am Dienst teilzunehmen ist daher nicht vom Direktionsrecht nach $\$ 106$ GewO gedeckt, sofern sie entgegen den Empfehlungen des Robert Koch-Instituts erfolgt. Es handelt sich schließlich um eine Weisung mit gesetzeswidrigem Inhalt, die der Mitarbeiter nicht befolgen muss ${ }^{51}$. In diesem Fall ist der Arbeitnehmer also nicht zur Aufnahme seiner Tätigkeit in der medizinischen Einrichtung verpflichtet.

\section{Homeoffice}

Um die Gefahr eines Ansteckungsrisikos zu reduzieren, äußern Arbeitnehmer gegenüber ihrem Arbeitgeber besonders in Zeiten der COVID-19-Pandemie vermehrt den Wunsch, im Homeoffice zu arbeiten. Zumindest für Mitarbeiter in der Krankenhausverwaltung besteht diese Möglichkeit. Ärztliches, pflegerisches sowie sonstiges medizinisches Personal kann hiervon verständlicherweise keinen Gebrauch machen ${ }^{52}$. Ein gesetzlicher Anspruch auf ein Arbeiten von zu Hause besteht für Arbeitnehmer ohnehin nicht, außer aus Tarif- oder Arbeitsvertrag sowie Betriebsvereinbarung folgt etwas anderes ${ }^{53}$. Allenfalls kann in absoluten Ausnahmesituationen die Schaffung eines Arbeitsplatzes im Homeoffice unter Bezugnahme auf die Rücksichtnahmepflicht des Arbeitgebers nach $\$ 241$ Abs. 2 BGB geboten $\operatorname{sein}^{54}$. Ob sich hieraus aber auch ein Anspruch auf Homeoffice zum Schutz der Gesundheit des Arbeitnehmers im Hinblick auf die Gefährdungslage durch das Corona-Virus SARS-CoV-2 ableiten lässt, bleibt mehr als fraglich. Daher verbleibt es bei den Arbeitsvertragsparteien, einvernehmlich eine entsprechende Vereinbarung für das Arbeiten im Homeoffice zu treffen. Gesteht der Arbeitgeber seinem Mitarbeiter ein Arbeiten von zu Hause aus zu, sollte er auch erforderliche Rahmenbedingungen, etwa zur Einhaltung von Arbeitsschutz- und Datenschutzbestimmungen, fixieren. Sofern die Initiative für ein Arbeiten im Homeoffice vom Arbeitgeber ausgeht, kann er dies gegenüber dem Arbeitnehmer nicht kraft seines Weisungsrechts nach $\$ 106$ GewO einseitig anordnen ${ }^{55}$. Allerdings finden sich bereits erste Stimmen in der rechtswissenschaftlichen Literatur, die die Weisung für eine Homeoffice-Pflicht in Anbetracht der durch das Corona-Virus hervorgerufenen außergewöhnlichen Umstände für gerechtfertigt halten ${ }^{56}$.

\section{Urlaubsplanung}

Vielfach verplant das Krankenhauspersonal nahezu seinen gesamten Jahresurlaub im Rahmen der Jahresurlaubsplanung für das jeweilige Kalenderjahr. Führt nunmehr die COVID-19-Pandemie dazu, dass ärztliches und pflegerisches Personal über einen sehr langen Zeitraum in der Klinik zur Patientenversorgung eingesetzt werden muss, stellt sich die Frage, ob der Arbeitgeber bereits genehmigten Urlaub widerrufen darf oder eine Urlaubssperre verhängen kann. Arbeitsrechtlich gilt der Grundsatz, dass bereits genehmigter Erholungsurlaub zu gewähren ist und nicht widerrufen werden $\mathrm{kann}^{57}$. Eine arbeitsvertragliche Klausel, die einem Krankenhausträger ein entsprechendes Recht einräumt, verstößt gegen zwingendes Urlaubsrecht $(\$ \$ 1,13 \mathrm{BUrlG})$ und ist damit rechtsunwirksam ${ }^{58}$. Gleiches gilt für eine tarifvertragliche Abrede zu Ungunsten des Arbeitnehmers ${ }^{59}$. Eine einvernehmliche Aufhebung der Urlaubserteilung ist jederzeit möglich ${ }^{60}$. Darüber hinaus kann der Krankenhausträger in besonderen unvorhersehbaren Ausnahme- und Notfällen berechtigt sein, bereits gewährten Urlaub zu widerrufen ${ }^{61}$. Führt die COVID-19-Pandemie zu einem gravierenden Personalmangel im Krankenhaus, ist der Widerruf bereits genehmigten Urlaubs - in dieser absoluten Ausnahmesituation - gerechtfertigt. Den betreffenden Mitarbeitern sind etwaig anfallende Stornokosten etc. zu erstatten. Der Widerruf eines gewährten Urlaubs aus dringenden betrieblichen Gründen ist mitbestimmungsfrei ${ }^{62}$. Zudem dürfte im Zuge der COVID-19-Pandemie auch die Anordnung einer Urlaubssperre gerechtfertigt sein.

\section{Kündigungsrecht}

\section{Diebstahl von Schutzartikeln und "Emmely-Entscheidung"}

Insbesondere in Zeiten der COVID-19-Pandemie verdient ärztliches sowie pflegerisches Personal höchste Anerkennung. Leider gibt es aber auch in dieser Berufsgruppe einige wenige (!) schwarze Schafe. Hiervon zeugt der vereinzelte Diebstahl von Atemschutzmasken, Desinfektionsmitteln und Einweg-

49) Hierzu Preis, in: ErfK, 20. Aufl. 2020, \$616 BGB, Rdnr. 13.

50) https://www.rki.de/DE/Content/InfAZ/N/Neuartiges_Coronavirus/Kontaktperson/Dokumente_Tab.html.

51) Griese in: Küttner, Personalbuch, 27. Aufl. 2020, Weisungsrecht, Rdnr. 16.

52) Etwas anderes mag noch für die radiologische Befundung gelten

53) LAG Rheinland-Pfalz, ArbRAktuell 2015, 158; Oberthür, MDR 2015, 1269

54) Näher dazu Isenhardt, DB 2016, 1499.

55) LAG Berlin-Brandenburg, NZA-RR 2019, 287.

56) Im Einzelnen Fuhlrott/Fischer, NZA 2020, 345, 349; ebenso mit anderem Ansatz Weller/Lieberknecht/Habrich, NJW 2020, 1017, die einen Anspruch auf Homeoffice in dieser Krisensituation sowohl auf Wunsch des Arbeitgebers als auch des Arbeitnehmers im Einzelfall für gerechtfertigt halten.

57) BAG, NZA-RR 2010, 473, 474; Spinner, in: MüKoBGB, 8. Aufl. 2020, §611a, Rdnr. 857.

58) BAG, NZA 2001, 100.

59) BAG, NZA 2001, 100, 102

60) LAG Hamm, NZA-RR 2003, 347.

61) BAG, NJW 1982, 2087, wonach ein einseitiger Widerruf der Urlaubserteilung durch den Arbeitgeber ,als Ausnahmefall nur bei ganz unvorhergesehenen Ereignissen " zulässig sei.

62) Wank, in: ErfK, 20. Aufl. 2020, \87 BetrVG, Rdnr. 47. 
handschuhen. In „normalen“ Zeiten wären auf derartige Delikte wohl die Grundsätze der „Emmely-Entscheidung“ des BAG anwendbar gewesen, wonach eine fristlose, verhaltensbedingte Kündigung wegen eines sog. Bagatelldelikts regelmäßig nicht gerechtfertigt ist ${ }^{63}$. Bei den vorbezeichneten medizinischen Schutzartikeln handelt es sich - umgangssprachlich ausgedrückt - um ,Pfennigartikel“"64, deren Diebstahl zwar eine Straftat darstellt, aber arbeitsrechtlich als Bagatelldelikt zu qualifizieren ist. Dieses Delikt berechtigt das Krankenhaus lediglich zum Ausspruch einer Abmahnung. Aber auch hier hat die COVID-19-Pandemie die Maßstäbe verschoben, sodass das Entwenden derartiger Schutzartikel anders zu beurteilen ist. Insbesondere die Nachfrage nach FFP2- oder FFP3-Schutzmasken - welche nach epidemiologischen Erkenntnissen auch dem jeweiligen Träger tatsächlich Schutz vor einer Corona-Infektion bieten ${ }^{65}$ - ist seit dem Auftreten des Corona-Virus SARS-CoV-2 deutlich angestiegen. Infolgedessen ist auch der Preis nahezu explodiert, mit der Folge, dass es sich bei der Mitnahme entsprechender Artikel nicht mehr um den Diebstahl eines geringwertigen Gegenstandes handelt. $\mathrm{Zu}-$ dem muss jedem Mitarbeiter im Gesundheitswesen bewusst sein, dass die Ausstattung eines Krankenhauses mit ausreichend Schutzartikeln im Rahmen der COVID-19-Pandemie elementare Voraussetzung für die Aufrechterhaltung des Geschäftsbetriebes ist. Anderenfalls würden sowohl Mitarbeiter, als auch Patienten einer nicht vertretbaren Gesundheitsgefährdung ausgesetzt (siehe Punkt VI.). Eine auBerordentliche verhaltensbedingte Kündigung - ohne vorherige Abmahnung - scheint daher durchaus gerechtfertigt.

\section{Diebstahl von Schutzartikeln und vorweggenommene Abmahnung}

Zudem haben viele Krankenhausleitungen ihre Belegschaft explizit auf die prekäre Versorgungslage mit Schutzartikeln hingewiesen und in Rundschreiben oder Bekanntmachungen mehrfach betont, dass die „Mitnahme“ derartiger Artikel kein Kavaliersdelikt ist, sondern eine Straftat, die mit allen zur Verfügung stehenden arbeitsrechtlichen Disziplinarmaßnahmen verfolgt wird. Plakativ kann man in diesem Zusammenhang von einer Zero-Tolerance-Politik sprechen, die viele Krankenhäuser hier fahren. Vor diesem Hintergrund stellt sich die Frage, ob derartige Bekanntmachungen als sog. vorweggenommene Abmahnung gewertet werden können. Eine solche liegt vor, wenn dem Arbeitnehmer die Vertragswidrigkeit seines Verhaltens bereits aus schriftlichen und mündlichen Äußerungen des Arbeitgebers bekannt war und er daher nicht lediglich mit der Erteilung einer (individuellen) Abmahnung rechnen durfte ${ }^{66}$. Das Rechtsinstitut der vorweggenommenen Abmahnung ist in der arbeitsgerichtlichen Spruchpraxis umstritten ${ }^{67}$. Betont werden immer die besonderen Umstände des Einzelfalles. Nach hier vertretener Ansicht sind jedenfalls die Bekanntmachungen der Krankenhausleitung zum Umgang mit Schutzartikeln, welche den expliziten Hinweis enthalten, dass der Diebstahl zwangsläufig die Kündigung zur Folge hat, als antizipierte Abmahnung zu werten ${ }^{68}$. In Zeiten der COVID-19-Pandemie sollte wirklich jedem Mitarbeiter - insbesondere im Krankenhaus - die Verwerflichkeit eines Schutzartikeldiebstahls bewusst sein.

\section{Arbeitsschutzmaßnahmen bei COVID-19-Pandemie}

\section{Biostoffverordnung}

Nach den $\int \$ 617$ bis 619 BGB sowie $\$ 4$ ArbSchG ist der Arbeitgeber - und im Besonderen ein Krankenhaus - verpflichtet, die bei ihm arbeitenden Menschen vor Gefahren für Leben und Gesundheit bei der Verrichtung ihrer Ar- beit $\mathrm{zu}$ schützen (Fürsorgepflicht). Bei Regelungen, die auf einen Schutz vor dem Corona-Virus SARS-CoV-2 gerichtet sind, hat der Betriebsrat grundsätzlich nach $₫ 87$ Abs. 1 Nr. 7 BetrVG (Gesundheitsschutz) ein Mitbestimmungsrecht. Bei Beschäftigten, die tätigkeitsbedingt mit dem Corona-Virus SARS-CoV-2 umgehen müssen - beispielsweise mit Proben im Labor oder pflegerischem und ärztlichem Personal, welches infizierte oder verdächtige Personen behandeln muss - richtet sich dieser Schutz vornehmlich nach der Biostoffverordnung ${ }^{69}$. Insbesondere hat das Krankenhaus die Belange des Arbeitsschutzes in Bezug auf Tätigkeiten mit Biostoffen in seine betriebliche Organisation einzubinden und hierfür die erforderlichen personellen, finanziellen und organisatorischen Voraussetzungen zu schaffen. Das Corona-Virus SARS-CoV-2 ist hierbei als biologischer Arbeitsstoff in die Risikogruppe 3 eingestuft. Nach $\$ 6$ Abs. 2 BioStoffV hat der Arbeitgeber im Rahmen einer Gefährdungsbeurteilung für alle gezielten Tätigkeiten mit biologischen Arbeitsstoffen die in Betracht kommenden Schutzmaßnahmen zu ermitteln. Hierbei sind immer mindestens die allgemeinen Hygienemaßnahmen der Schutzstufe 1 nach Anhang II oder III der BioStoffV festzulegen. Darüber hinaus sind für biologische Arbeitsstoffe der Risikogruppe 3 die Sicherheitsmaßnahmen der Schutzstufe 3 zu treffen. Nach $\ 10$ Abs. 5 BioStoff V darf der Arbeitgeber Beschäftigten gezielte Tätigkeiten mit biologischen Arbeitsstoffen der Risikogruppe 3 nur übertragen, sofern diese ausreichend fachkundig und eingewiesen sind. Entsprechend des Beschlusses des Ausschusses für Biologische Arbeitsstoffe (ABAS) vom 19.2. 2020 gilt für diagnostische Laboratorien ein gestuftes Verfahren. Bei Ärzten und pflegerischen Mitarbeitern ist der Krankenhausträger zur Vermeidung von Infizierungen grundsätzlich verpflichtet, angemessene persönliche Schutzausrüstung (sog. PSA) zur Verfügung zu stellen. Hierzu gehören insbesondere Schutzhandschuhe sowie Atemschutzmasken, zumindest Mundschutz. Dies gilt auch für die Patienten zum Schutz der Mitarbeiter. Nach den Empfehlungen des Robert Koch-Instituts besteht bei Einhaltung der empfohlenen Schutzmaßnahmen auch kein Anlass für eine Absonderung oder regelmäßige Untersuchungen asymptomatischer Mitarbeitender des medizinischen Personals auf das Corona-Virus SARS$\mathrm{CoV}-2^{70}$.

\section{Zurückbehaltungsrecht an der Arbeitsleistung}

Die Behandlung von infizierten Patienten ist ohne entsprechende Ausrüstung nicht zumutbar. Die Mitarbeiter sind demnach auch nicht verpflichtet ihre Arbeitsleistung zu erbringen. Ihnen steht sogar ein Zurückbehaltungsrecht an der Arbeitsleistung nach $\$ 273$ Abs. 1 BGB zu, wenn der Krankenhausträger die ihn treffende Pflicht zur Gewährleistung der Sicherheit am Arbeitsplatz (\$ 618 Abs. 1 BGB; $\$ 4$ ArbSchG; Vorgaben der Biostoffverordnung) nicht erfüllt ${ }^{71}$. Nach in der Literatur vertretener

63) BAG, NZA 2010, 1227.

64) Vgl. nur die Ruhr Nachrichten v. 29.3.2020

65) https://www.aerzteblatt.de/nachrichten/111423/BAeK-Praesident-ruft-zum-Tragen-von-Schutzmasken-auf.

66) LAG Bad.-Württ., NZA-RR 2011, 76, 79.

67) Bejahend: BAG, NZA 2001, 893; ablehnend: LAG Berlin-Brandenburg, NZA-RR 2012, 353, 355.

68) So auch Schuster/Darsow, NZA 2005, 273, 277; Mengel/Hagemeister, BB 2007, 1386, 1392.

69) Verordnung über Sicherheit und Gesundheitsschutz bei Tätigkeiten mit Biologischen Arbeitsstoffen (Biostoffverordnung).

70) https://www.rki.de/DE/Content/InfAZ/N/Neuartiges_Coronavirus/Kontaktperson/Dokumente_Tab.html.

71) BAG, NZA 1997, 86. 
Ansicht, setzt das Zurückbehaltungsrecht nach $₫ 273$ Abs. 1 BGB keine unmittelbare Gefahr für Leben oder Gesundheit voraus. Gleichwohl soll bei geringfügigen oder kurzfristigen Verstößen des Arbeitgebers gegen Arbeitsschutzpflichten, die keinen nachhaltigen Schaden beim Mitarbeiter bewirken können, kein Zurückbehaltungsrecht ausgeübt werden können ${ }^{72}$. Das Risiko sich mit dem Corona-Virus SARS-CoV-2 zu infizieren ist ein unmittelbares Lebens- bzw. Gesundheitsrisiko, sodass ein unzureichender Schutz hiergegen das Krankenhauspersonal zu einem Zurückbehaltungsrecht nach $\$ 273$ Abs. 1 BGB berechtigt ${ }^{73}$. Falls der Arbeitnehmer tatsächlich von seinem Zurückbehaltungsrecht Gebrauch macht - und zur Ausübung dieses Rechts berechtigt ist -, gerät der Arbeitgeber in Annahmeverzug, mit der Folge, dass der Arbeitnehmer seines Vergütungsanspruchs nicht verlustig wird ${ }^{74}$.

\section{Fazit}

Die relativ plötzlich auftretende COVID-19-Pandemie stellt die Akteure im deutschen Gesundheitswesen vor große Herausforderungen. Insbesondere Krankenhäuser waren zu Beginn der Pandemie gezwungen - zunächst vornehmlich aufgrund der behördlichen Vorgabe elektive Eingriffe abzusagen -, ihre Personaleinsatzplanung von heute auf morgen zu ändern. Nahezu jedes Krankenhaus hat sein Schichtsystem auf ein 12-Stunden-Schichtsystem umgestellt und eine Kohortierung durchgeführt, um bei der Versorgung von SARS-CoV-2-infizierten Patienten eine organisatorische und räumliche Trennung des zugewiesenen Personals zu erreichen. Derartige Maßnahmen sind nach den Ausnahmeregelungen der \$\$14, 15 ArbZG zulässig. Sie unterliegen zudem der Mitbestimmung des Betriebsrates nach $₫ 87$ Abs. 1 Nr. 2 BetrVG. Am Corona-
Virus SARS-CoV-2 erkrankte Mitarbeiter medizinischer Einrichtungen haben Anspruch auf Entgeltfortzahlung nach $\$ 3$ Abs. 3 EFZG. Krankenhauspersonal, welches unter amtlich angeordneter Quarantäne steht oder dem sogenannten beruflichen Beschäftigungsverbot nach dem Infektionsschutzgesetz unterliegt, ist von seiner Arbeitsverpflichtung befreit. Hier greift der Entschädigungsanspruch gegenüber dem Staat nach $\$ 56$ Abs. 1 IfSG. Um jedoch den effektiven Einsatz medizinischen Personals - verständlicherweise auch nach dem Kontakt mit einem SARS-CoV-2-infizierten Patienten - zu ermöglichen, hat das Robert Koch-Institut Empfehlungen zum Management von Kontaktpersonen veröffentlicht. Diese Empfehlungen verdeutlichen, wie wichtig die Einhaltung der Arbeitsschutzmaßnahmen im Zuge der COVID-19-Pandemie ist. Kommt der Krankenhausträger diesen Maßnahmen nicht oder nicht ausreichend nach, steht dem Mitarbeiter ein Zurückbehaltungsrecht an seiner Arbeitsleistung nach $\$ 273$ Abs. 1 BGB zu. Elementare Voraussetzung, damit ein Krankenhaus seine Arbeitsschutzmaßnahmen erfüllen kann, ist jedoch die ausreichende Ausstattung mit Schutzausrüstung wie Atemschutzmasken, Desinfektionsmitteln, Einweghandschuhen sowie Schutzkitteln. Bedauerlicherweise ist die Versorgung mit diesen Materialien - entgegen anderslautender politischer Verlautbarungen - alles andere als gewährleistet.

72) Blomeyer, in: MHdB ArbR, 4. Aufl. 2018, \41, Rdnrn. 15, 16; Reinfeld, in: MAH ArbR, 4. Aufl. 2017, \$34, Rdnr. 13.

73) Allerdings ist wichtig zu betonen, dass auch im Falle einer drohenden Infizierungsgefahr die Arbeitspflicht nach wie vor fortbesteht. Das bestehende Risiko einer Infizierung rechtfertigt es nicht, die Arbeitsleistung zurückzuhalten - nur die unzureichenden Schutzmaßnahmen.

74) Reinfeld, in: MAH ArbR, 4. Aufl. 2017, §34, Rdnr. 13.

\section{Rechtliche Betreuung in Zeiten der COVID-19 Pandemie}

\section{Anna Schwedler und Sarah Glaab}

\begin{abstract}
Ältere Menschen und Menschen mit Vorerkrankungen bedürfen eines besonderen Schutzes vor einer Infektion mit COVID-19. Dieser Schutz gestaltet sich letztlich so, dass sich die Menschen in das Private zurückziehen und den Kontakt $\mathrm{zu}$ Anderen weitgehend meiden sollen. Allerdings können vor allem ältere Menschen eine besondere Unterstïtzung bei Erledigung ihrer Angelegenheiten benötigen, die im Hinblick auf die Teilhabe im Rechtsverkehr häufig durch eine rechtliche Betreuung sichergestellt wird. Dafür ist grundsätzlich der persönliche Kontakt zwischen den Betroffenen, den Betreuern, den Betreuungsgerichten, den Sachverständigen und den Verfahrenspflegern erforderlich. Es fragt sich daher, welche Auswirkungen die Maßnahmen zur Eindämmung des Coronavirus auf das betreuungsrechtliche Verfahren und das bestehende Betreuungsverhältnis haben.
\end{abstract}

Dr. iur. Anna Schwedler und Ass. iur. Sarah Glaab,

Institut für Zivilrecht und Wirtschaftsrecht,

Fachbereich Rechtswissenschaft,

Goethe-Universität Frankfurt,

Theodor-W.-Adorno-Platz 4,

60629 Frankfurt (Postfach 29), Deutschland

\section{Das Verfahren der Betreuerbestellung}

Die landesrechtlichen Maßnahmen zur Eindämmung der COVID-19 Pandemie scheinen insbesondere die Betreuungsgerichte vor neue Herausforderungen zu stellen, da die verfahrensrechtlichen Regelungen vor allem den persönlichen Kontakt zwischen den Beteiligten voraussetzen. Aufgrund der Auswirkungen auf das Selbstbestimmungsrecht des Betroffenen sind spezielle prozessuale Sicherungen vorgesehen, wie Beteiligungsbefugnisse, Anhörungsrechte und eine Pflicht zur sachverständigen Begutachtung, die allesamt mit persönlichen Interaktionen verbunden sind.

Im Vordergrund des Betreuungsverfahrens steht die Prüfung ob bzw. in welchem Umfang der Betroffene seine Angelegenheiten tatsächlich nicht mehr selbst oder durch Bevollmächtigte besorgen kann. Eine Betreuung darf schließlich nur dann eingerichtet werden, wenn und soweit sie erforderlich ist, vgl. \1896 BGB. Anhand dieses Erforderlichkeitskriteriums lässt sich auch die Ausgestaltung des Betreuungsverfahrens erklären, das eine Reihe von Verfahrensvorschriften vorsieht, um die Rechte des Betroffenen zu wahren. 